\title{
Diffraction of light by acoustic waves in liquids
}

\author{
K. A. I. L.Wijewardena Gamalath, G. L. A. U Jayawardena \\ Department of Physics, University of Colombo, Colombo 03, Sri Lanka \\ E-mail address: imalie@phys.cmb.ac.lk
}

\begin{abstract}
For the acusto-optic interactions in liquids, an equation for the diffraction light intensity was obtained in terms of Klein Cook parameter $Q$. With optimized parameters for $Q$, incident light wave length of $\lambda=633 \mathrm{~nm}$, sound wave length of $\Lambda=0.1 \mathrm{~mm}$, acusto-optic interaction length $L=0.1 \mathrm{~m}$, and refractive index of the liquid in the range of 1 to 2, the existence of ideal Raman-Nath and Bragg diffractions were investigated in terms of phase delay and incident angle. The ideal Raman-Nath diffraction slightly deviated when the Klein Cook parameter was increased from 0 to 1 for low phase delay values and for large phase delay, the characteristics of the Bessel function disappeared. Higher value of Klein Cook parameter gave Bragg diffraction and ideal Bragg diffraction was obtained for $Q$ $\sim 100$. A slight variation of the incident angle from Bragg angle had a considerable effect on Bragg diffraction pattern. Klein Cook parameter with the change of acoustic wave frequency was investigated for liquids with refractive index in the range1.3-1.7 and their diffraction patterns were compared with practically applicable acusto-optic crystals. For acusto-optic diffractions in liquids, sound velocity plays an important role in Bragg regime with $Q$ increasing with increasing acoustic frequency. As acoustic wave frequency exceeded $10 \mathrm{MHz}$ most of the liquids reached Bragg regime before these crystals.
\end{abstract}

\section{Keywords:}

Acusto-optic interactions; Raman-Nath and Bragg diffraction; Klein Cook parameter; Phase delay

\section{INTRODUCTION}

An acoustic signal injected into the medium by means of piezoelectric transducer, produces regions of compression and rarefaction as it propagates through the medium. The induce strain, changes the index of refraction of the medium and an optical beam passing through this medium may be deflected or modulated and frequency shifted. Light diffracting on the inhomogeneties of the refractive index known as acusto-optic effect forms in the far field a salient diffraction pattern. In 1922 Brillouin $^{1}$ predicted that a liquid traversed by compression waves of short wavelength irradiated by visible light give rise to a diffraction phenomenon similar to that of a grating. Raman and Nath ${ }^{2}$ designed a general ideal model of interaction in 1936. This model was developed by Phariseau ${ }^{3}$ for the first order diffraction. In 1967 Klein and $\mathrm{Cook}^{4}$ introduce a parameter known as quality factor $Q$, a normalized measure of grating thickness to distinguish the two diffraction regimes. Moharam and Young ${ }^{5}$ showed that a parameter $\rho$, independent of the grating thickness first defined by Nath is an effective replacement for $Q$. However, because the index of modulation must be large for a thin grating to produce appreciable diffraction, thin enough gratings tend to operate in the Raman-Nath regime. 
In this study, for the diffraction of light by acoustic waves in liquids, an equation for the diffracted light intensities was obtained in terms of Klein Cook parameter using time varying permittivity in Maxwell's equations. As acusto-optic interaction is determined by light-sound geometry, optical and acoustic properties of the acoustic material, the variation of diffraction pattern with the change of the Klein Cook parameter for different wave lengths of the incident light, wavelengths of acoustic wave, widths of acoustic beam and acoustic mediums were investigated. The ratio of intensity of diffracted light with respect to the Klein Cook parameter $Q$ and phase delay for an isotropic interaction with no change in polarization with the assumption that there was no optical loss in the acoustic medium were plotted in 2-D for these optimum parameters. The conditions that give Raman-Natha and Bragg diffraction patterns were studied by varying the, phase angle, Klein-Cook parameter and incident angle.

The maximum Klein Cook parameters and diffraction intensities of some liquids with the change of acoustic wave frequency were investigated for liquids with refractive indices in the range 1.3 and 1.7. These were compared with practically applicable acusto-optic crystals. The diffraction pattern for acetic acid with a Klein Cook parameter of $Q=11$ is presented. The Numerical solutions of the output light intensities were obtained using Runge-Kutta routine with control error.

\section{RAMAN-NATH AND BRAG DIFFRACTION REGIMES}

Acusto-optic interaction can be described as a diffraction of an optical wave by a travelling phase grating induced by an acoustic wave ${ }^{1}$. As the phase grating Doppler-shifts the optical frequency, it can be used to deflect, modulate, or filter the optical beam. These interactions based on photoelastic effect are either isotropic or anisotropic, depending on the optical properties of the acusto-optic medium. Isotropic acusto-optic interactions which do not change the polarization of optical beam can result either multiple or single diffracted optical orders. The Raman-Nath regime is observed at relatively low acoustic frequencies at small acusto-optic interaction lengths. This type of diffraction takes place at an arbitrary incident angle of light roughly normal to the acoustic beam and the diffraction pattern contains many diffraction orders (Figure 1(a)) of symmetrically distributed light intensity given by Bessel functions. By contrast, the Bragg regime is observed at high acoustic frequencies usually exceeding $100 \mathrm{MHz}$. The diffraction pattern of this regime even at large acoustic power consists of two diffraction maxima of zero

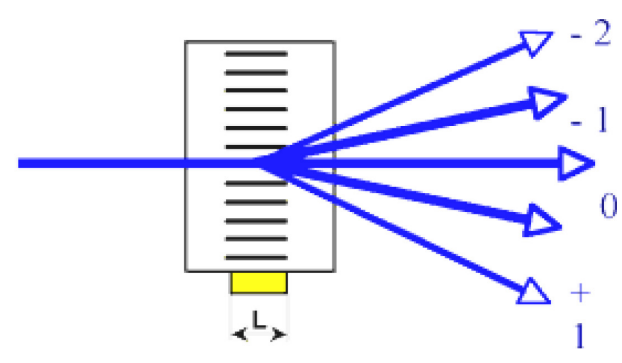

(a)

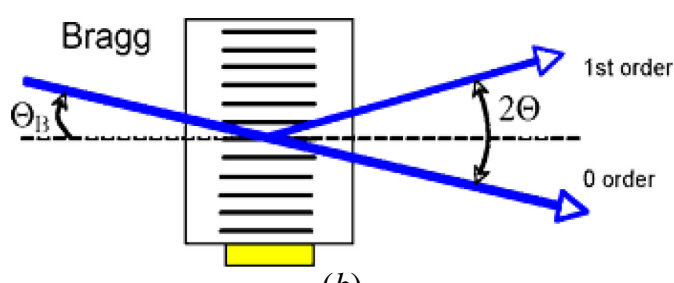

(b)

Figure 1. (a) Raman-Nath diffraction regime $(b)$ Bragg diffraction regime and first orders (Figure 1(b)). At Bragg angle of incidence $\Theta_{B}$ only one diffraction order is produced while others are annihilated by destructive interference.

This single-order isotropic Bragg diffraction is much more efficient and therefore is widely used in practical devices. There is no well-defined boundary between these two diffraction regimes. 
As the injected acoustic signal propagates through the medium producing regions of compression and rarefactions, the produced strain changes the optical properties of the medium due to changes in the index of refraction of the medium. The strain-induced changes in the optical properties of the acusto-optic medium may lead to rotation of the light polarization. The optical changes studied through the index ellipsoid by calculating the difference of the ellipsoid equations for the unperturbed and perturbed states, result an expression for the change in the refractive index $n_{i j}$ in terms of photoelastic fourth rank tensor $p_{i j k l}$ and the strain components $S_{k l}$

$$
\Delta n_{i j}=\frac{1}{2} n_{i j}^{3} p_{i j k l} S_{k l} \quad(i, j, k, l=1,2,3)
$$

For optically isotropic materials, the coefficients $p_{i j k l}$ are symmetrical with respect to $i j$ and $k l$ indices and can be contracted to $p_{m n}(m, n=1,2, \ldots, 6)$. The mathematical description of the photoelastic effect depending on the directional properties of acusto-optic material requires a tensor relation between the elastic strain and photoelastic coefficient given by the change in the dielectric permeability $\Delta B_{i j}$

$$
\Delta B_{i j}=\Delta\left(\frac{1}{n^{2}}\right)_{i j}=p_{i j k l} S_{k l} \quad(i, j, k, l=1,2,3)
$$

The pressure in the sound wave creates a travelling wave of rarefaction and compression, which in turn create perturbations in the index of refraction. Thus, the acoustooptic devices may be thought as a thin phase grating with an effective grating line separation equal to the wavelength of the sound in the acoustic medium. The phase grating splits the incident light into various diffracted orders and the directions of diffracted or scattered light inside the sound cell are governed by the grating equation,

$$
\sin \phi_{m}=\sin \phi_{i n c}+m \lambda_{0} / \Lambda \quad(m=0, \pm 1, \pm 2, . .)
$$

where $\phi_{m}$ is the angle of $m^{\text {th }}$ order diffracted light beam, $\phi_{i n c}$ the angle of incidence, $\lambda_{0}$ the wavelength of light and $\Lambda$ is the wavelength of the sound measured in the acoustic medium. The phase-grating approach of acousto-optic interaction is a somewhat an over simplification.

The interaction of sound and light can be considered as collision of photons and phonons. In order for these particles to have well-defined momenta and energies, the interaction must assumed to be of monochromatic plane waves of light and sound with sufficiently wide width $L$ of the transducer to produce plane wave front at a single frequency. If the plane wave vectors of incident light, diffracted light and sound in acoustic medium are denoted by $k_{0}, k_{ \pm 1}$ and $K$, respectively, then the condition for conservation of momentum can be written as

$$
k_{ \pm 1}=k_{0} \pm K
$$

where plus sign indicate the upshifted (Figure 2) and minus sign, the downshifted diffraction (Figure 3). Since for all practical cases $K|<<| k_{0}$ the magnitude of $k_{ \pm 1}$ is essentially equal to $k_{0}$. 


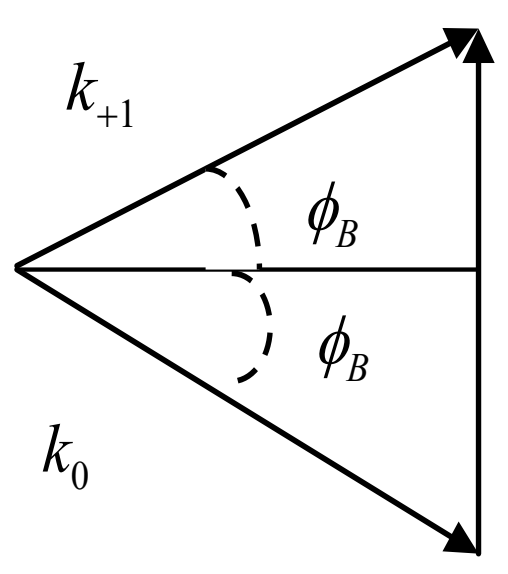

(a)

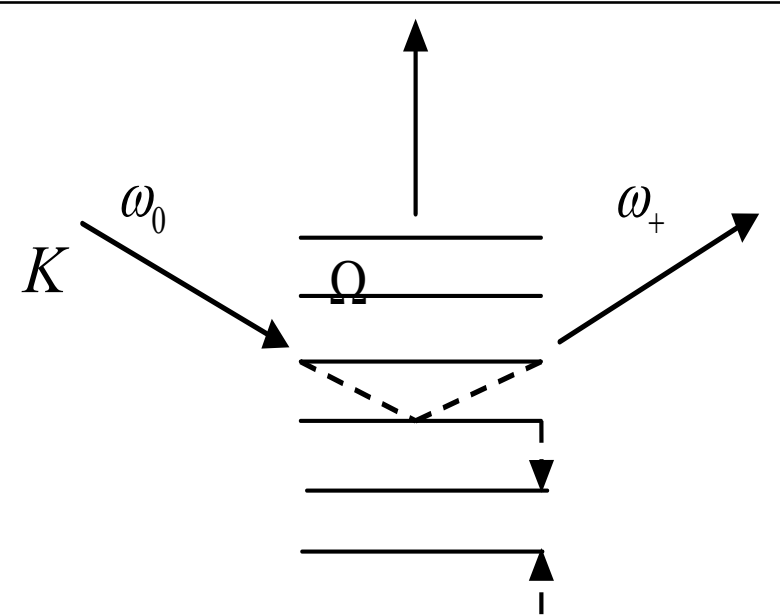

(b)

Figure 2. (a) wave vector diagram, $(b)$ experimental configuration for upshifted interaction

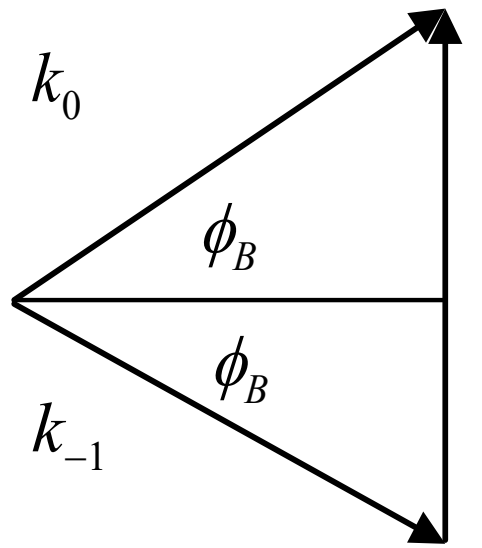

(a)

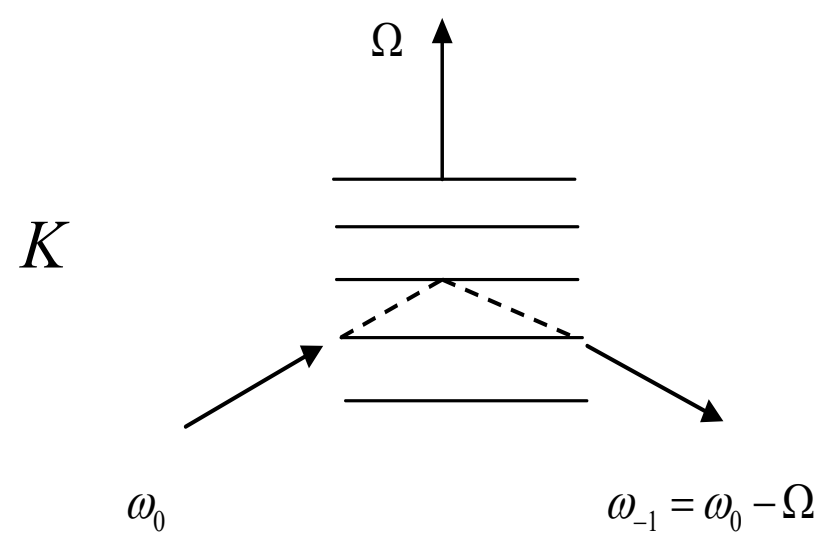

(b)

Figure 3. (a) wave vector diagram, $(b)$ experimental configuration for downshifted interaction

Therefore the wave vector triangle shown in Figure 2 and 3 are nearly isosceles. The corresponding equation for conversation of energy is

$$
\omega_{ \pm 1}=\omega_{0} \pm \Omega
$$

where $\omega_{0}, \Omega$ and $\omega_{ \pm 1}$ are the frequencies of the incident light, sound and scattered light respectively. Positive signs in equation 4 and 5 refer to phonon absorption while negative signs refer to stimulated phonon emission.

As the width $L$ of the transducer decreases the sound waves spread out over angles $\pm \Lambda / L$. For the upshifted interaction, the $K$-vector can be oriented through an angle $\pm \Lambda / L$ due to the spreading of sound (Figure 4). In order to have only one diffracted order $\left(k_{+1}\right)$ of light generated, the condition 


$$
\frac{\lambda_{0}}{\Lambda}>>\frac{\Lambda}{L} \quad \text { or } \quad L>>\frac{\Lambda^{2}}{\lambda_{0}}
$$

has to be imposed. An acusto-optic modulator satisfying this condition is operating in the Bragg regime and the device is a Bragg cell.

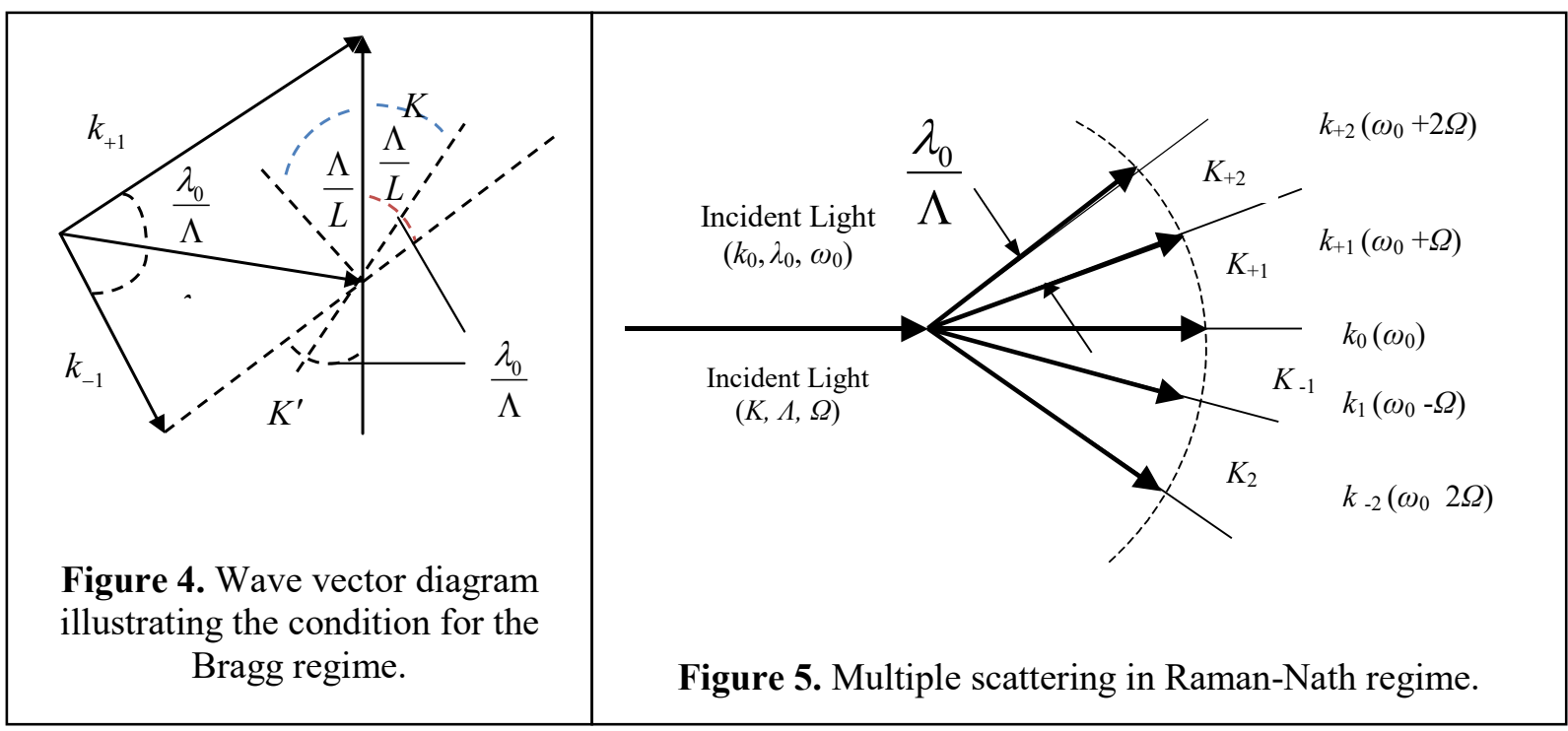

When $L$ is sufficiently short, $\mathrm{L}<<\Lambda^{2} / \lambda_{0}$ second regime of diffraction (scattering), called Raman-Nath (or Debye-Sears) occurs. In this regime positive $k_{+1}$ and negative $k_{-1}$ first order scattered light are generated simultaneously. These may interact with the sound field again to produce higher orders of diffracted light. In this re-scattering process called strong interaction, scattered light in the Bragg regime, may re-scatter back into the zeroth order. In the Raman-Nath regime, many orders may exist due to the availability of plane waves of sound at various angles (figure 5). For example, $k_{+1}$ is generated due to diffraction of $k_{0}$ by $K_{+1}$ while $k_{+2}$ is generated through the diffraction of $k_{+1}$ by $K_{+2}$.

$K_{ \pm p}^{s}(p=0, \pm 1, \pm 2, \ldots)$ denote the appropriate components of the plane wave spectrum of the sound. The requirement of conservation of energy leads to the equation

$$
\omega_{m}=\omega_{0}+m \Omega
$$

where $\omega_{m}$ is the frequency of the $m$-the order scattered light.

\section{ACUSTO-OPTIC EFFECT}

The interaction between the optical fields $E_{0}(\boldsymbol{r}, t)$ and sound field $S(\boldsymbol{r}, t)$ can be described by Maxwell's equations. If the interaction takes place in an optically inhomogeneous, nonmagnetic, isotropic medium, characterized by permeability $\mu_{0}$ and a permittivity $\tilde{\varepsilon}(\boldsymbol{r}, t)$, then a source-free optical field incident on the time-varying permittivity representing the action of the sound field can be written as ${ }^{6}$ 


$$
\tilde{\varepsilon}(\boldsymbol{r}, t)=\varepsilon+\varepsilon^{\prime}(\boldsymbol{r}, t)=\varepsilon C S(\boldsymbol{r}, t)
$$

where $C$ is a proportional constant dependent on the medium. Maxwell's equations in SI units read

$\nabla \cdot D=\rho_{v}, \quad \nabla \cdot B=0, \quad \nabla \times E=-\frac{\partial B}{\partial t}, \quad \nabla \times H=J=J_{c}+\frac{\partial D}{\partial t}$

where $E$ and $H$ are the electric and magnetic field strengths, $D$ and $B$ are the electric and magnetic flux densities and $\rho_{v}$ and $J_{c}$ are the charge and current densities respectively. For a two-dimensional sound field in $x-z$ plane with $E$ polarized along the $y$-direction, one can show that $E . \nabla \tilde{\varepsilon}=0$. Since sound frequency is much lower than the light frequency, the time variation of $\tilde{\varepsilon}(\boldsymbol{r}, t)$ is much slower than $E(\boldsymbol{r}, t)$ and Maxwell's equation lead to

$$
\nabla^{2} E(\boldsymbol{r}, t)-\mu_{0} \varepsilon \frac{\partial^{2} E(\boldsymbol{r}, t)}{\partial t^{2}}=\mu_{0} \varepsilon^{\prime}(\boldsymbol{r}, t) \frac{\partial^{2} E(\boldsymbol{r}, t)}{\partial t^{2}}
$$

Harmonic variations are introduced in the incident light and sound in the forms

$$
\begin{gathered}
E_{i n c}(\boldsymbol{r}, t)=\operatorname{Re}\left[\mathrm{E}_{i n c}(\boldsymbol{r}) e^{j \omega_{0} t}\right]=\frac{1}{2}\left(\mathrm{E}_{i n c}(\boldsymbol{r}) e^{j \omega_{0} t}+\mathrm{E}_{i n c}^{*}(\boldsymbol{r}) e^{-j \omega_{0} t}\right) \\
\frac{\varepsilon^{\prime}(\boldsymbol{r}, t)}{\varepsilon}=\operatorname{Re}\left[C S(\boldsymbol{r}) e^{j \Omega t}\right]=\frac{1}{2}\left(C S(\boldsymbol{r}) e^{j \Omega t}+C S^{*}(\boldsymbol{r}) e^{-j \Omega t}\right)
\end{gathered}
$$

where $E_{\text {inc }}{ }^{*}$ and $S^{*}$ denotes the complex conjugate. Equation 12 follows from equation 8 . The total field due to harmonic frequency mixing is

$$
E(\boldsymbol{r}, t)=\frac{1}{2} \sum_{m=-\infty}^{\infty} \mathrm{E}_{m}(\boldsymbol{r}) \exp \left[j\left(\omega_{0}+m \Omega\right) t\right]+\mathrm{E}_{m}{ }^{*}(\boldsymbol{r}) \exp \left[-j\left(\omega_{0}+m \Omega\right) t\right]
$$

With equation 12 and 13 and the assumption $\Omega<<\omega_{0}$ equation 10 gives an infinite coupleddifferential equation

$$
\nabla^{2} \mathrm{E}_{m}(\boldsymbol{r})+k_{0}^{2} \mathrm{E}_{m}(\boldsymbol{r})+\frac{1}{2} k_{0}^{2} C S(\boldsymbol{r}) \mathrm{E}_{m-1}(\boldsymbol{r})+\frac{1}{2} k_{0}^{2} C S^{*}(\boldsymbol{r}) \mathrm{E}_{m+1}(\boldsymbol{r})=0
$$


where $k_{0}=\omega_{0} \sqrt{\mu_{0} \varepsilon}$ is the propagation constant of light in the medium and $\mathrm{E}_{m}(\boldsymbol{r})$ is the amplitude of the $m^{\text {th }}$ order light at frequency $\omega_{0}+m \Omega$.

In a conventional interaction configuration for a uniform sound wave of finite width $L$ propagating along $x$ direction (Figure 6), the sound phasor is represented by

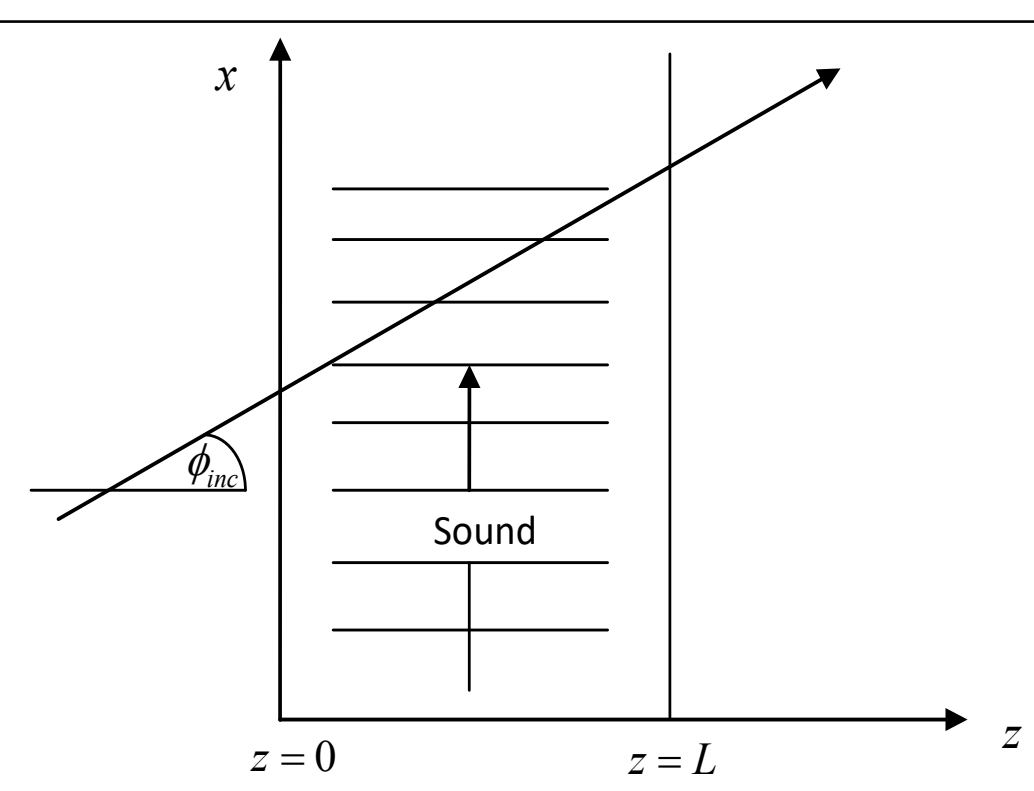

Figure 6. Sharp boundary configuration

$$
S(\boldsymbol{r})=S(z, x)=A e^{-j k x}
$$

where $A$ in general can be complex $A=|A| e^{j \theta}$. A plane sound wave propagating along $x$ direction is given by equation 12 as $\widetilde{\varepsilon} \sim C|A| \cos (\Omega t-K x+\theta)$. The incident plane wave of light can be represented as

$$
\mathrm{E}_{i n c}(\boldsymbol{r})=\psi_{i n c} \exp \left(-j k_{0} z \cos \phi_{i n c}-j k_{0} x \sin \phi_{i n c}\right)
$$

where $\phi_{i n c}$ is the incident angle of light. In $\mathrm{z}-\mathrm{x}$ plane we seek $\mathrm{E}_{m}(r)$ in the form

$$
\mathrm{E}_{m}(\boldsymbol{r})=\mathrm{E}_{m}(z, x)=\psi_{m}(z, x) \exp \left(-j k_{0} z \cos \phi_{m}-j k_{0} x \sin \phi_{m}\right)
$$

With the choice for $\phi_{m}$ given by equation 3 ,

$$
\sin \phi_{m}=\sin \phi_{i n c}+m \lambda_{0} / \Lambda=\sin \phi_{i n c} K / k_{0}
$$

where $k_{0}=2 \pi / \lambda_{0}$ is the wave number of light inside the acoustic medium and $K=2 \pi / \Lambda$ is the wave number of sound. Assuming $\psi_{m}$ is a slowly varying function of $z$ so that within a wavelength of light $\partial \psi_{m} / \partial z$ does not change appreciably, $\partial^{2} \psi_{m} / \partial z^{2}$ can be neglected compared to $\left(2 j k_{0} \cos \phi_{m}\right) \partial \psi_{m} / \partial z$ and $\omega_{0}>>m \Omega$. In addition $\phi_{m}$ is very small quantity $\left(\phi_{m}<<1\right)$ and we assume $\psi_{m}(z, x) \approx \psi_{m}(z)$. With these physical conditions equation 14 reduces to 
$\frac{d \psi_{m}}{d z}=-j \frac{k_{0} C A}{4 \cos \phi_{m}} \psi_{m-1} e^{-j k_{0} z\left(\cos \phi_{m-1}-\cos \phi_{m}\right)}-j \frac{k_{0} C A^{*}}{4 \cos \phi_{m}} \psi_{m+1} e^{-j k_{0} z\left(\cos \phi_{m+1}-\cos \phi_{m}\right)}$

This equation can be solved for the boundary condition $\psi_{m}=\psi_{i n c} \delta_{m 0}$ at $\quad z \leq 0$ where $\delta_{m 0}$ is the Kronecker delta function. This equation is one variant of the well-known RamanNath equation ${ }^{2}$.

The Raman-Nath equation was reformulated into a set of equations by Korpel and Poon $^{7}$. For a planer acoustic wave of sinusoidal travelling wave, refractive index $n(r, t)$ can be described by ${ }^{8}$

$$
n(r, t)=n_{0}+\Delta n \sin (\Omega t-K r)
$$

where $n_{0}$ is the average refractive index of the medium, $\Delta n$ is the amplitude of the refractive index change due to the acoustic strain, and $K, \Omega$ are the wave number and frequency of acoustic wave respectively. Then $\tilde{\varepsilon}(\boldsymbol{r}, t)$ can be written as

$$
\widetilde{\mathcal{E}}(r, t)=\varepsilon_{0} n^{2}(r, t)=\varepsilon_{0}\left[n_{0}+\Delta n(r, t)\right]^{2} \simeq \varepsilon_{0} n_{0}^{2}\left[1+\frac{2 \Delta n(r, t)}{n_{0}}\right]
$$

From equation 12:

$$
C S(\boldsymbol{r}, t)=\frac{2 \Delta n(\boldsymbol{r}, t)}{n_{0}} \text { or } \quad C|S|=C|A|=2 \frac{(\Delta n)_{\max }}{n_{0}}
$$

where $(\Delta n)_{\max }$ denotes the peak amplitude of the assumed harmonic variation of $\Delta n(r, t)$. Assuming that $A$ is real and non-negative, the phase delay $\alpha$ of the light through the acoustic medium is

$$
\alpha=\frac{k_{0} C|A| L}{2}=\left(\frac{k_{0}}{n_{0}}\right)(\Delta n)_{\max } L
$$

where $L$ is the acusto-optic interaction length along the direction of propagation of light. The normalized distance inside the acusto-optic cell is $\xi=z / L$ with $\xi=1$ signifying the exit plane of the cell. For small values of $K / k_{0}$, the phase terms of equation 19 can be expanded in a power series by using equation 18 ,

$$
k_{0} z\left(\cos \phi_{m \mp 1}-\cos \phi_{m}\right)= \pm k_{0} z\left[\left(\frac{K}{k_{0}}\right) \sin \phi_{i n c}+\left(m \mp \frac{1}{2}\right)\left(\frac{K}{k_{0}}\right)^{2}+\ldots \ldots . .\right]
$$


The acusto-optic interaction geometries in which an appreciable amount of light is transferred out of zero order into diffracted orders can be examined by using the Kelin and Cook parameter $^{4} Q$,

$$
Q=\frac{K^{2} L}{k_{0}}=\frac{2 \pi \lambda L}{n_{0} \Lambda^{2}}
$$

where $\Lambda$ is the acoustic wavelength and $\lambda$ is the incident light wavelength in air. Using first order approximations given in equation 21 to 24 , equation 19 can be re-written as

$$
\left.\frac{d \psi_{m}}{d \xi}=-j \frac{\alpha}{2} e^{-j \frac{1}{2} Q \xi\left(\frac{\phi_{i n c}}{\phi_{B}}+(2 m-1)\right.}\right)_{\psi_{m-1}}-j \frac{\alpha}{2} e^{j \frac{1}{2} Q \xi\left(\frac{\phi_{i n c}}{\phi_{B}}+(2 m+1)\right)} \psi_{m+1}
$$

where $\psi_{m}=\psi_{i n c} \delta_{m 0}$ at $\xi=0$ with small angle approximation $\sin \theta \approx \theta, \cos \theta \approx 1$, or $\phi_{m}<<1$. Equation 26 is a special case of general plane wave multiple scattering theory ${ }^{7,9}$ valid for any sound field. Raman-Nath regime is defined by the condition that $L<<\Lambda^{2} / \lambda_{0}$ or $Q<<1$. For this criterion, the phase terms in the exponents involving $Q(m-1 / 2)$ and $Q(m+1 / 2)$ can be neglected if $m Q<<1$. The condition $Q<<1$ is more stringent for higher diffracted orders $(m)$. For oblique incidence $\left(\phi_{i n c} \neq 0\right)$ equation 26 read

$$
\frac{d \psi_{m}}{d \xi}=-j \frac{\alpha}{2} e^{-j \frac{1}{2} Q \xi \frac{\phi_{i n c}}{\phi_{B}}} \psi_{m-1}-j \frac{\alpha}{2} e^{j \frac{1}{2} Q \xi \frac{\phi_{i n c}}{\phi_{B}}} \psi_{m+1}
$$

and for normal incidence $\left(\phi_{i n c}=0\right)$ :

$$
\frac{d \psi_{m}}{d \xi}=-j \frac{\alpha}{2}\left(\psi_{m-1}+\psi_{m+1}\right) \Rightarrow \psi_{m}=(-j)^{m} \psi_{i n c} J_{m}(\alpha \xi)
$$

the amplitude of the various scattered orders give Raman-Nath solution.

The Bragg diffraction is characterized by generation of two scattered orders. For downshifted interaction of Bragg angle of incidence $\left(\phi_{i n c}=\phi_{B}\right)$, the diffracted orders are 0 and -1 , whereas for upshifted interaction $\left(\phi_{i n c}=-\phi_{B}\right)$ orders are 0 and 1 . From equation 26, the coupled equations describing the downshifted and upshifted interaction are obtained as

$$
\frac{d \psi_{0}}{d z}=-j \frac{\alpha}{2} \psi_{\mp 1}, \quad \frac{d \psi_{\mp 1}}{d z}=-j \frac{\alpha}{2} \psi_{0}
$$


When $\cos \phi_{-1}=\cos \phi_{0}$ phase synchronism exist between the zeroth and the first orders. Hence $\phi_{0}=\phi_{B}=\phi_{i n c}, \quad \phi_{-1}=-\phi_{B}=-\phi_{i n c}$ and the zeroth and the first orders propagate symmetrically with respect to the sound wave front. The solutions for downshifted or upshifted interaction with the boundary conditions $\psi_{m}=\psi_{i n c} \delta_{m 0}$ and $z \leq 0$

$$
\psi_{0}=\psi_{i n c} \cos (\alpha \xi / 2), \quad \psi_{\mp 1}=-j \psi_{i n c} \sin (\alpha \xi / 2)
$$

give the scattered light for Bragg diffraction. The Bragg diffraction maxima appear only at Bragg angle $\phi_{B}$. In an optically isotropic medium for Bragg diffraction $\left|k_{i}\right| \simeq\left|k_{d}\right|$, the magnitude of the acoustic wave vector must satisfy the equation,

$$
|K|=2|k| \sin \phi_{B} \Rightarrow \sin \phi_{B}=\frac{\lambda}{2 n_{0} \Lambda}
$$

where $K=2 \pi / \Lambda, k=2 \pi / \lambda_{0}$ and $\lambda_{0}=\lambda / n_{0}$

\section{INTENSITY OF DIFFRACTION PATTERN WITH PHASE DELAY}

For the diffraction of light by acoustic waves in liquids, the Klein Cook parameter $Q$ given in equation 25 was analysed to obtain the optimum parameters. As acusto-optic interaction length $L$ is proportional to Klein Cook parameter, for longer interaction lengths, the

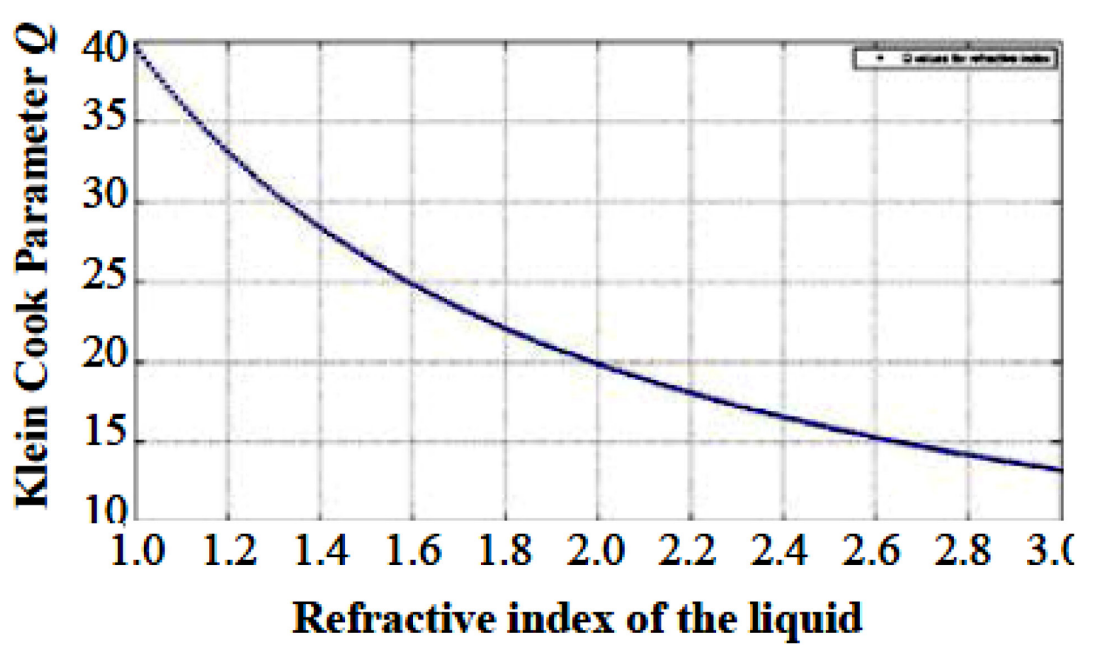

Figure 7. Graph of $Q$ vs. refractive index for $\lambda=633 \mathrm{~nm}, \Lambda=0.1 \mathrm{~mm}$ and

$$
L=0.1 \mathrm{~m}
$$

possibility of finding the diffraction pattern in Bragg diffraction regime in a given medium is higher. But in practice, the incident light and acoustic wave must not attenuate in the acustooptic medium.

As for the acusto-optic interaction length of $0.1 \mathrm{~m}$, the incident light intensity before and after the interaction was approximately equal, this value was used as acusto-optic interaction length. The acoustic wave length $\Lambda$ is inversely proportional to $Q$ and its range in water ${ }^{10}$ is $\Lambda=7.5 \mathrm{~cm}$ to $2.5 \times 10^{-4} \mathrm{~cm}$. Therefore the acoustic wavelength range used was $0.001 \mathrm{~mm}$ to $100 \mathrm{~mm}$. Although for higher values of light wavelength $\lambda$, there is a higher probability of finding the diffraction pattern in Bragg regime, for the visible light wavelength range $380 \mathrm{~nm}$ 
to $750 \mathrm{~nm}$, the highest value of Klein Cook parameter was obtained for the incident light wavelength of $633 \mathrm{~nm}$. By setting the incident light wavelength at $633 \mathrm{~nm}$, acusto-optic interaction length at $0.1 \mathrm{~m}$ and acoustic wavelength at $0.1 \mathrm{~mm}$ the parameter $Q$ was plotted with refractive index (figure 7).

From this graph the range between 1and 2 was selected for the refractive index. By defining $\phi_{i n c}=-(1+\delta) \phi_{B}$ where $\delta$ represents the deviation of the incident plane wave from the Bragg angle, equation 26 can be written as

$\frac{d \psi_{m}}{d \xi}=-j \frac{\alpha}{2} e^{-j \frac{1}{2} Q \xi(-(1+\delta)+(2 m-1))} \psi_{m-1}-j \frac{\alpha}{2} e^{j \frac{1}{2} Q \xi(-(1+\delta)+(2 m+1))} \psi_{m+1}$

In Raman-Nath diffraction, the incidence light is normal to the acoustic wave. An assigned incidence angle $\phi_{i n c}=0$ gives $\delta=-1$ and for ideal Bragg diffraction $\phi_{i n c}=\phi_{B}$, $\delta=0$. To investigate the diffraction of light by acoustic wave, upshifted diffracted order $-6 \leq m \leq 6$ was restricted to 13 and a set of 13 coupled equations were written out.

These set of equations were numerically solved by using Runge-kutta method with the assumption that acusto-optic interaction does not change the intensity of incident light before and after. A two dimensional form was considered as the height of the transducer was neglected and the number of modes included was increased until no further significant effect occurred for a given value of Klein Cook parameter.

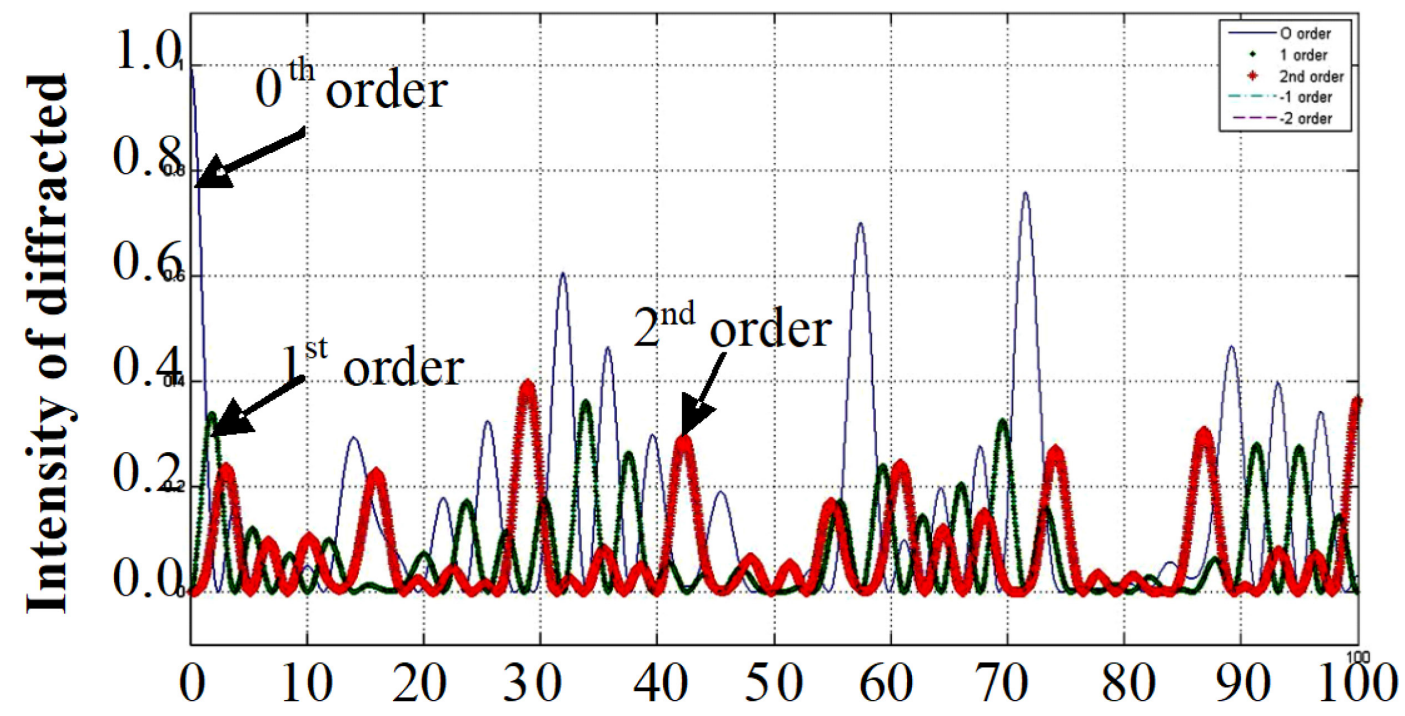

Peak phase delay

Figure 8. Intensity of diffracted orders vs. peak phase delay in the ideal Raman-Nath regime $(Q=0$ and $\delta=-1$ ) for normal incidence. 


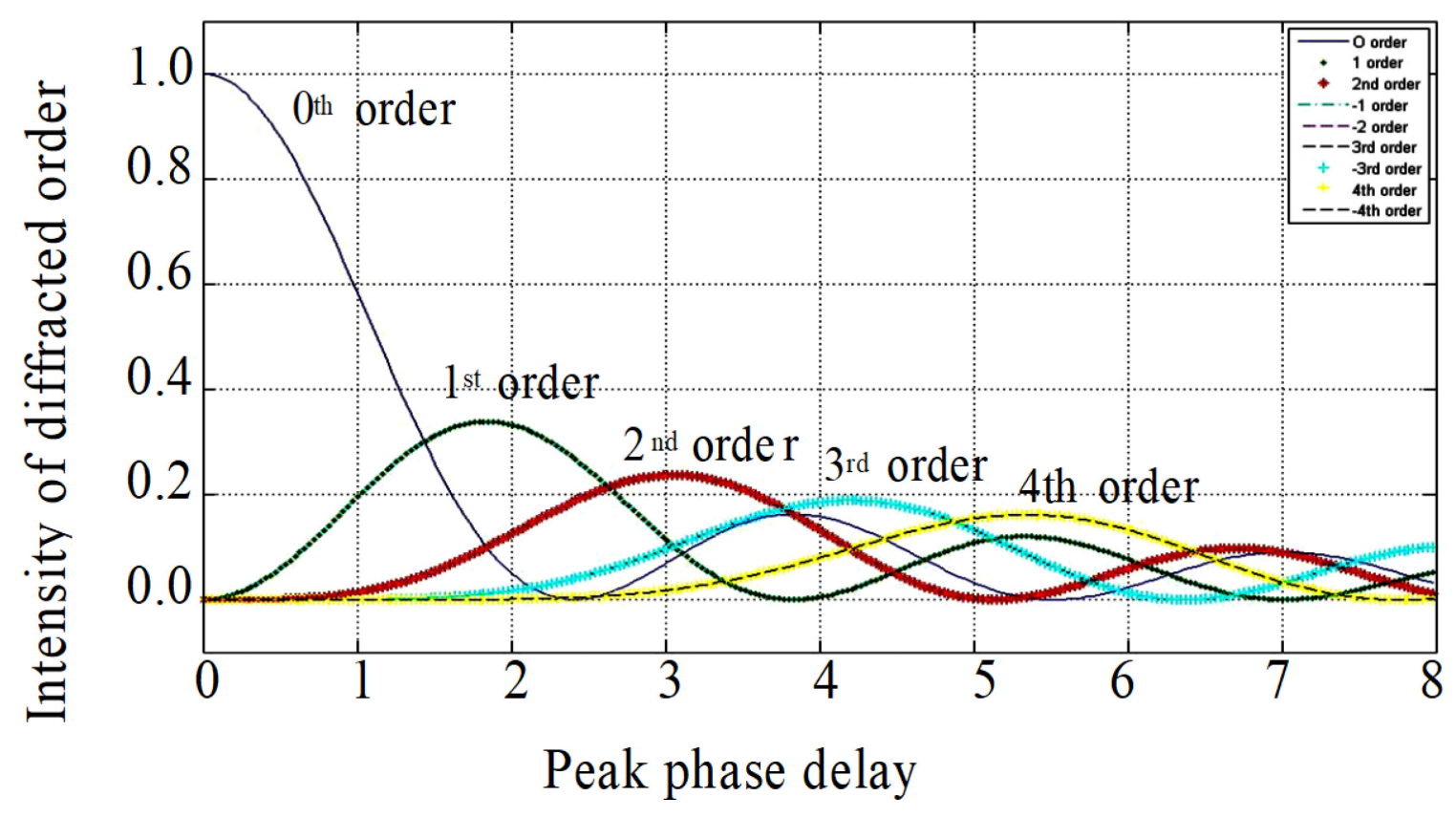

Figure 9. Intensity of diffracted orders vs. peak phase delay in the ideal Raman-Nath regime $(\mathrm{Q}=0$ and $\delta=-1) 8$ modes of negative and positive orders are overlapped at normal incidence.

The intensity ratio of diffracted orders $\left|\psi_{m}\right|^{2} /\left|\psi_{\text {inc }}\right|^{2}$ with phase delay $\alpha$ is shown in figure 8 for ideal Raman-Nath regime for normal incidence of light. When $Q=0$ either acusto-optic wave width must go to zero $(L \rightarrow 0)$ or refractive index of the acusto-optic medium should go to infinity $\left(n_{0} \rightarrow \infty\right)$. A medium satisfying either one of these condition gives ideal Raman-Nath diffraction. In practice since neither of these conditions can be satisfied, $Q=0$ is a non-physical situation. For the ideal Raman-Nath case, the sum of the intensities of the 13 modes (orders) was close to unity, the intensities of all other modes being negligibly small. The true zeros, for $Q=0$ which are the characteristic of the Bessel functions for ideal case, disappear for large value of phase delay $\alpha$.

Figure 9 shows the intensity of higher order interaction. Although there are several orders in the Raman-Nath diffraction regime, only four are presented. Various scattered orders for $Q=0$, illustrates the Bessel function dependence on phase angle for low phase delay. Since for normal incidence same magnitude orders of upshifted and downshifted waves have equal intensities, eight modes of negative and positive orders overlapped. Most of the total energy is transferred into zeroth order and the rest is transferred successively into large number of modes with intensity decreasing with increasing order. By setting, $\delta=0$ the diffraction pattern for Bragg angle of incidence was obtained for ideal Raman-Nath regime. There was no effect when the angle of incidence was changed from normal to Bragg angle of incidence for ideal Raman-Nath diffraction. The parameter $Q$ for Raman-Nath diffraction pattern was changed by stepwise for normal incidence of light. These are shown in Figure 10. When the parameter $Q$ was increased from 0 to 1 for low phase delay values, diffraction pattern began slightly to deviate from the ideal Raman-Nath diffraction. For non ideal RamanNath diffraction $Q=10$, the zero and first order intensities share the most of the energy of the incident light as indicated in the Figure 10. 

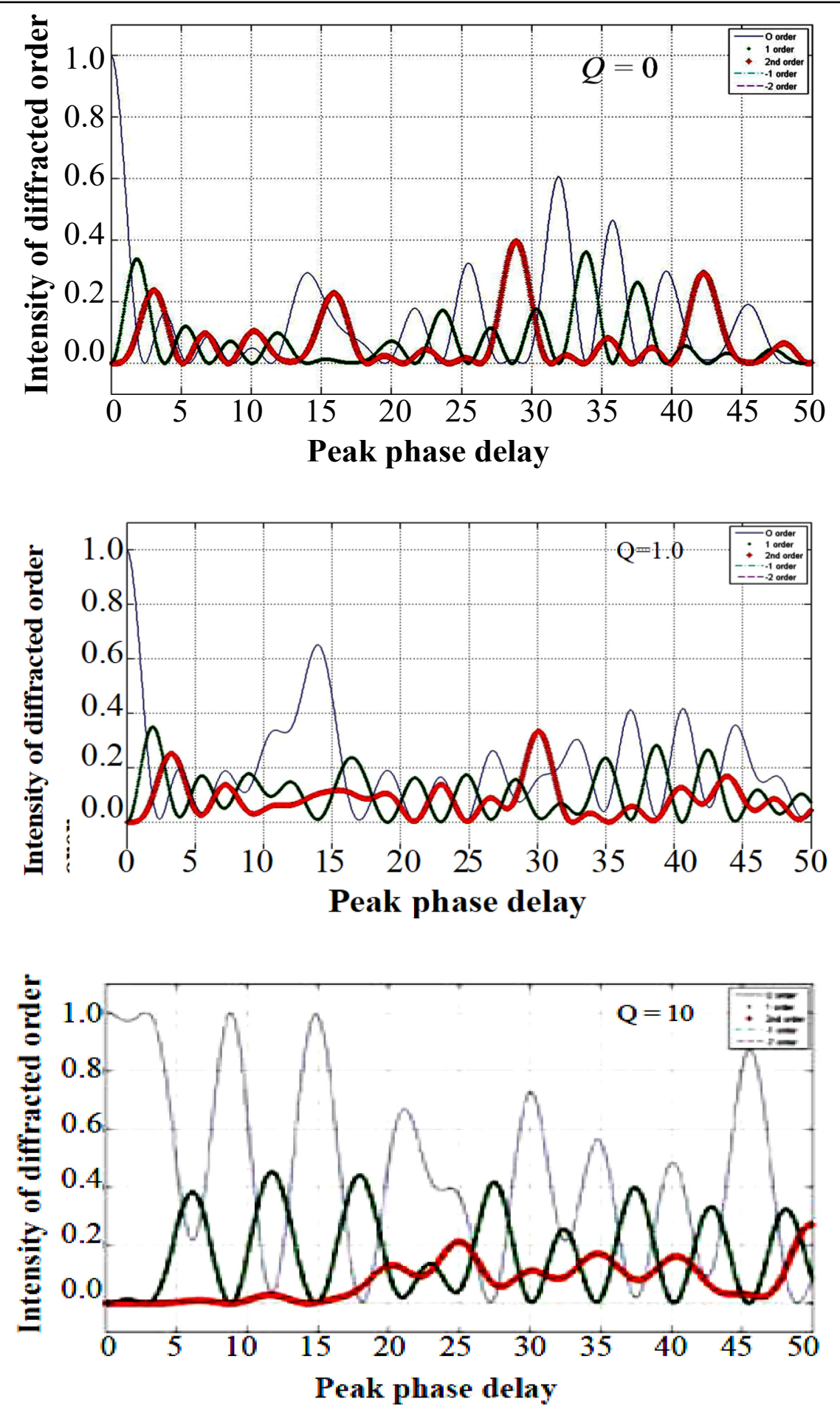

Figure 10. Intensity of diffracted orders vs. peak phase delay $(\alpha) \delta=-1$ for $Q=0,1$ (near the ideal Raman-Nath) and 10 (non ideal Raman-Nath)

The Bragg diffraction is established only when the incident light angle is equal to the Bragg angle. $\delta=0$ corresponds to the case of exact Bragg incidence for upshifted interaction defined by $\phi_{i n c}=-(1+\delta) \phi_{B}$. Figure 11 shows the intensity of diffracted orders near Bragg diffraction. In this case, periodic power exchange between the two main orders was no longer 
complete for larger value of phase delay indicating the presence of higher orders. For Bragg diffraction there were no overlaps of orders like in Raman-Nath regime. Ideal Bragg diffraction can be approximated at $Q=100$ for low phase delay values $(\alpha)$ (Figure 12 ).
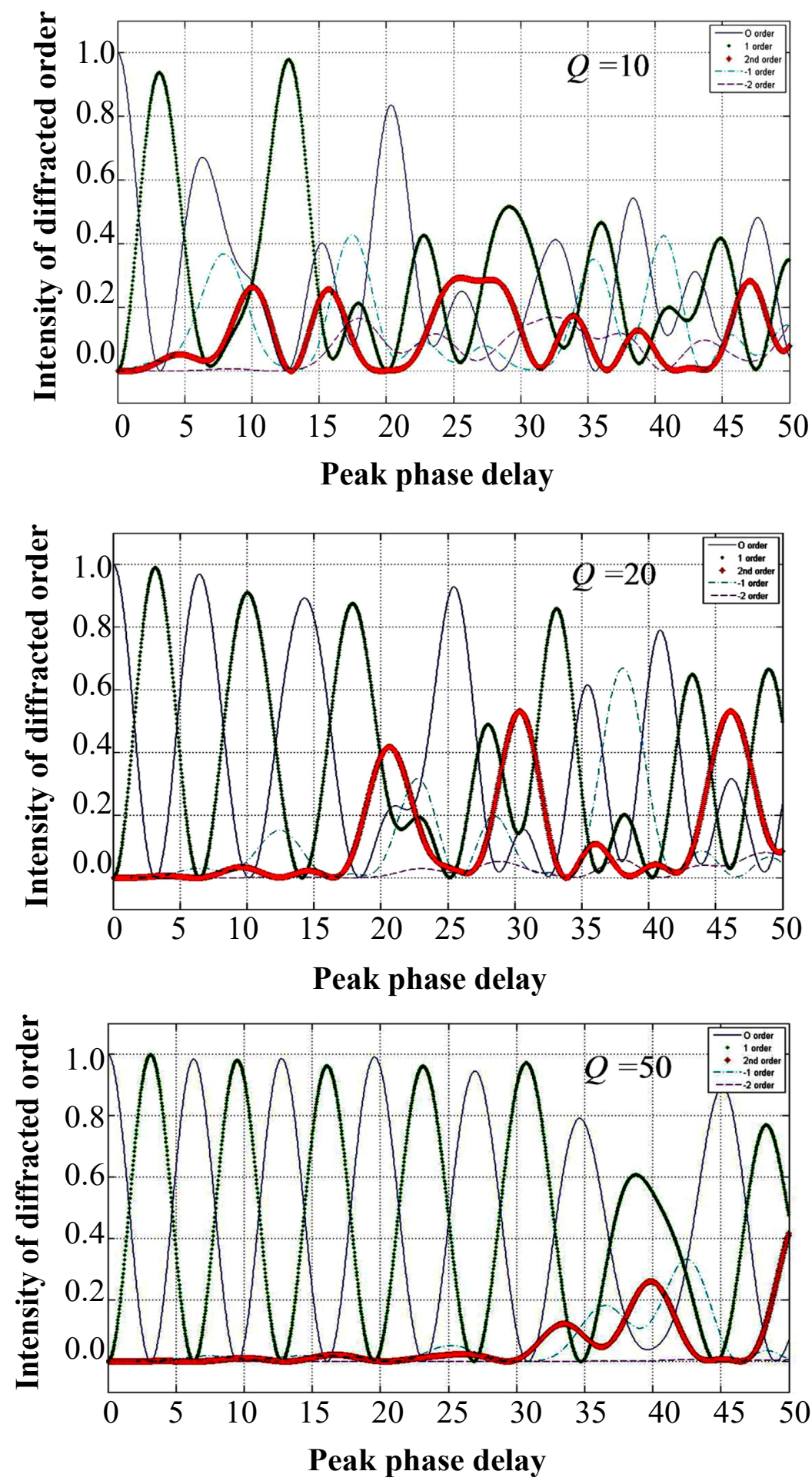

Figure 11. Intensity of diffracted orders vs. peak phase delay $(\alpha)$ for $\delta=0$ for $Q=10,20$ and 50 near-Bragg diffraction.

In this approximated region, a complete exchange of power between the zero and first order occur and higher orders are not present. For $Q=100$, the phase angle was slightly varied 
from zero. These are shown in figure 12. A deviation of the incidence angle from Bragg angle result an incomplete energy exchange between first two orders. The variation of incidence angle from Bragg angle has a considerable effect on diffraction of light when the diffraction regime was in Bragg regime.
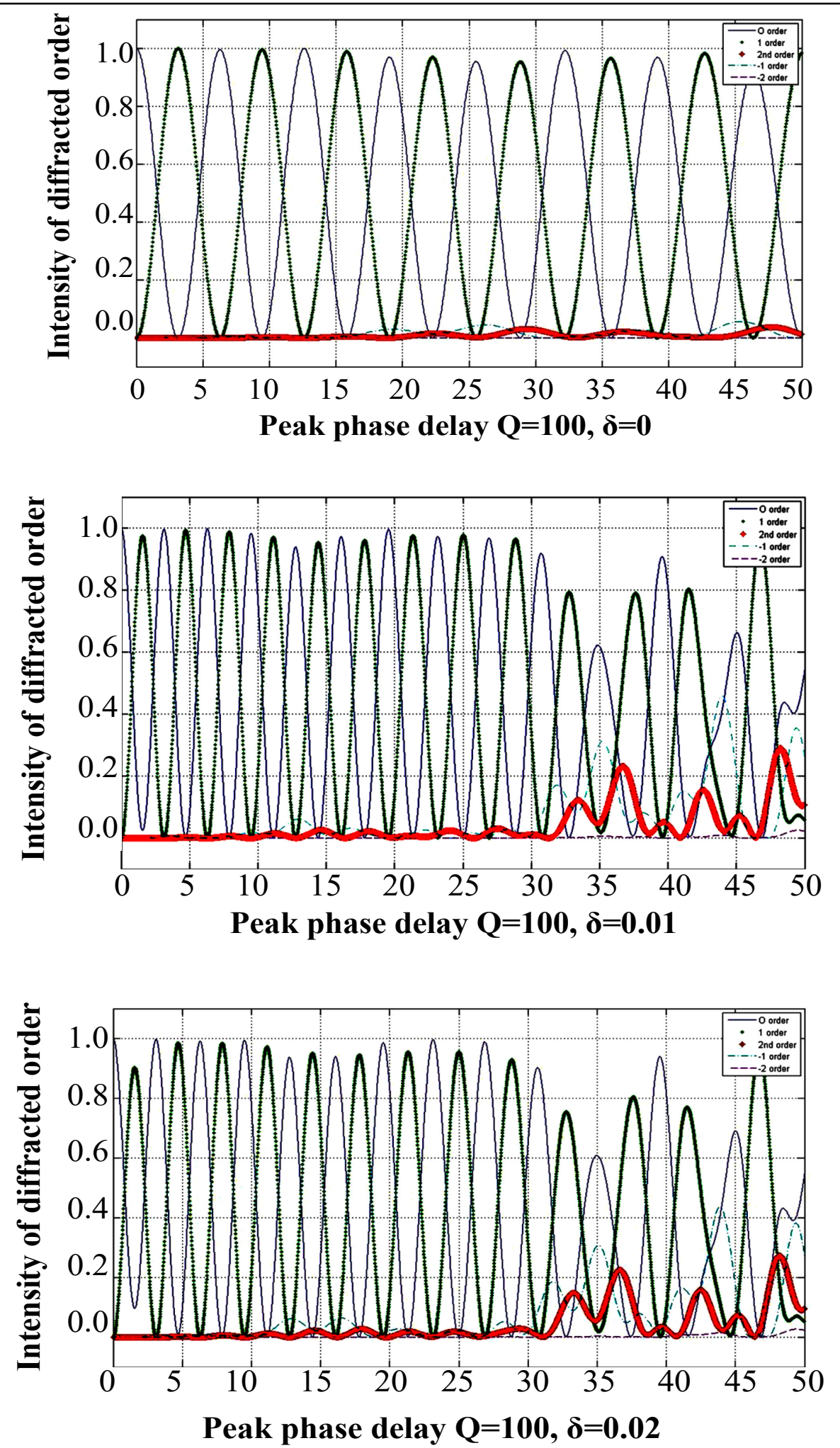

Figure 12. Intensity of diffracted orders vs. peak phase delay $(\alpha)$ near-Bragg diffraction for $Q=$ 100 , and for $\delta=0,0.01$ and 0.02 


\section{ACUSTO-OPTIC DIFFRACTION IN LIQUIDS}

Acusto-optic diffraction properties for different liquids were obtained by calculating Klein-Cook parameter for different sound frequencies. Refractive index range for these liquids was between 1.3 and 1.7. Fixing the incident light wavelength at 633 $\mathrm{nm}$ and acusto-optic interaction length at $0.1 \mathrm{~m}$, the parameter $Q$ for different acoustic wave frequencies were plotted for different liquids (Figure 13). The physical properties of these liquids are tabulated in the table 1 in order of decreasing value of maximum $Q$. Carbon tetrachloride had the largest $Q$ value whereas water had a low $Q$ value and ethylene glycol

Table 1. Refractive indices and sound velocities of fluids in the order of maximum $Q$ parameter

\section{Fluid}

Carbon tetrachloride

Ether

Chloroform

Heptanes

Alcohol, ethyl (ethanol)

Hexane

Carbon disulfide

Turpentine (wood)

Benzene

Water

Acetic Acid

Ethylene glycol

$\begin{array}{cc}\begin{array}{c}\text { Refractive } \\ \text { Index }\left(n_{0}\right)\end{array} & \begin{array}{c}\text { Sound } \\ \text { velocity }\left(\mathrm{ms}^{-1}\right) \\ 1.46\end{array} \\ 926 \\ 1.35 & 985 \\ 1.44 & 995 \\ 1.38 & 1138 \\ 1.36 & 1144 \\ 1.37 & 1203 \\ 1.63 & 1149 \\ 1.47 & 1240 \\ 1.50 & 1298 \\ 1.33 & 1493 \\ 1.37 & 1584 \\ 1.43 & 1644\end{array}$

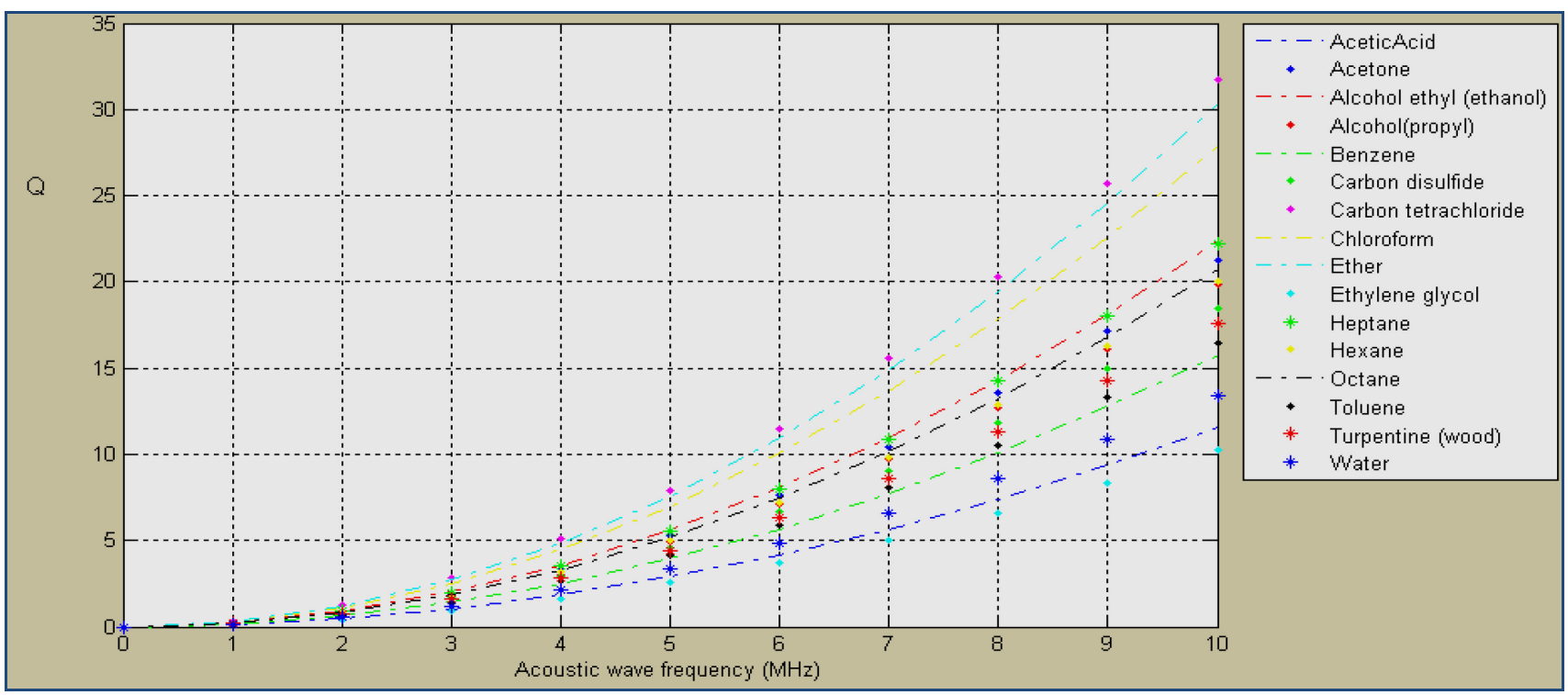

Figure 13. Graph of the parameter Q vs. Acoustic wave frequency liquids in table 1, with incident light wave length $\lambda=633 \mathrm{~nm}$ and acusto-optic interaction length $L=0.1 \mathrm{~m}$

the lowest $Q$ value for acusto-optic interaction. In practical use of liquids as acusto-optic interaction mediums although the refractive index is not an important physical property acusto-optic propagation velocity is an important parameter. Generally, a medium having lower sound velocity approach maximum parameter $Q$ faster but for carbon disulfide, a higher refractive index has more effect on $Q$ than sound wave velocity. Also heptane and ethanol have nearly equal $Q$ values. Liquids tabulated in table 1 gave various intensity patterns for physical parameters. Figure 14 shows the intensity variation of diffracted orders for acetic acid. The Klein Cook parameter was approximately equal to 11. 
The diffraction pattern was in the Bragg diffraction regime for the peak phase delay range of 0 to 5 . In order to have a long phase delay range for the Bragg diffraction pattern, $Q$ should have a higher value and this can be accomplished by increasing the acoustic wave frequency. The Bragg angle calculated for the acetic acid was $0.08356^{\circ}$.

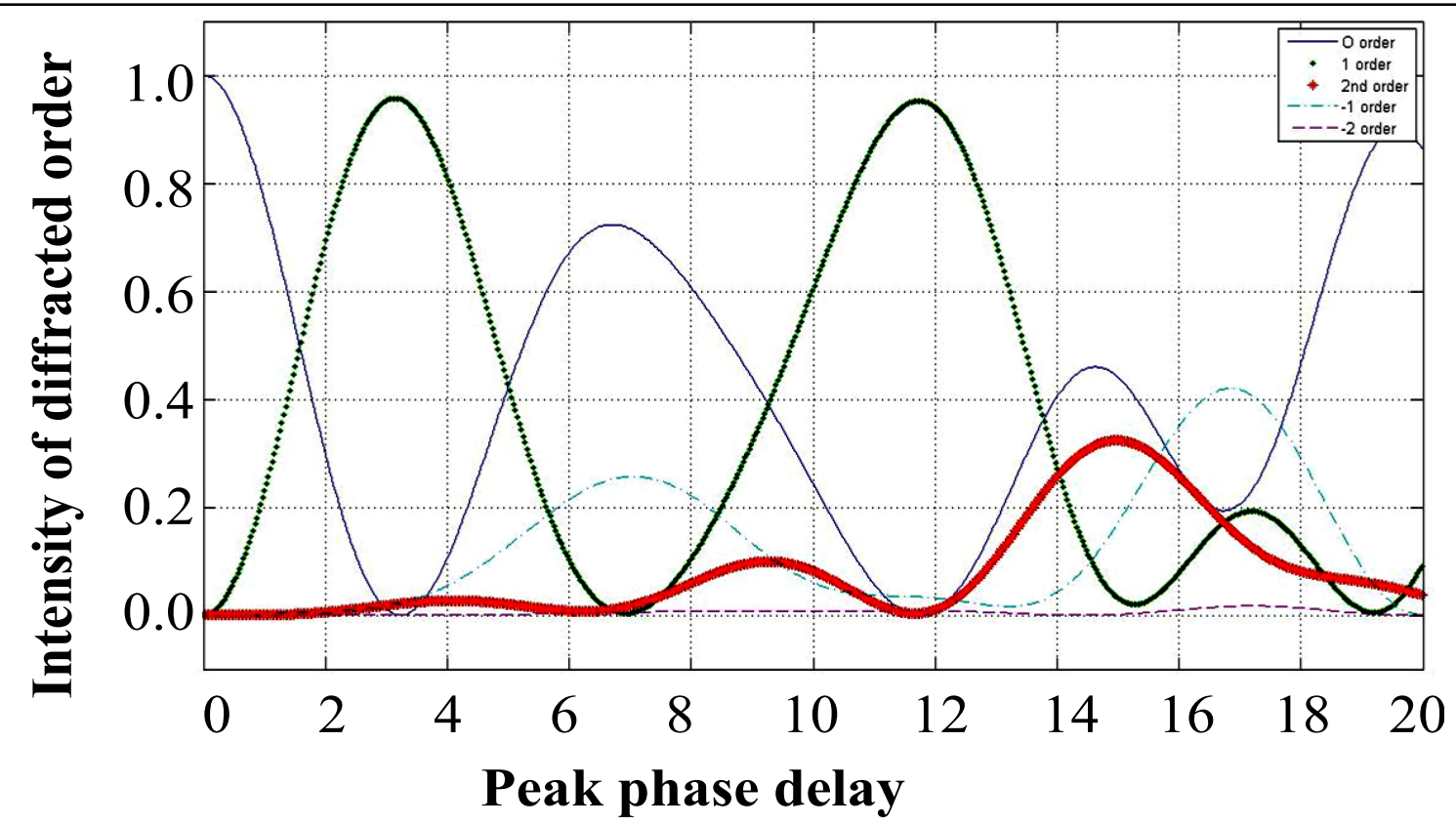

Figure 14. Intensities of diffracted orders vs. peak phase delay $(\alpha)$ for Acetic acid with the act of incident light with $\lambda=633 \mathrm{~nm}$, acoustic wave frequency $f=10 \mathrm{MHz}$ and acoustic wave width $L=0.1 \mathrm{~m}$

In practical applications of acusto-optic modulators, deflectors and tuneable filters, crystals are used as acoustic wave medium. The properties of materials used in acusto-optic devices are tabulated in table 2.

The parameter $Q$ for the liquids in table 1 and crystals in table 2 with respect to acoustic wave frequency were plotted in figure 15. Most of the liquids have higher value for Klein Cook parameter than the crystals except for $\mathrm{TeO}_{2}$ which has the lowest sound velocity.

In the Bragg diffraction regime, the liquids are more efficient than acusto-optic crystals other than $\mathrm{TeO}_{2}$. As acoustic wave frequency exceeded $10 \mathrm{MHz}$ most of the liquids reached Bragg regime before these crystals.
Table 2. Properties of Selected acusto-optic Materials $^{8}$

\begin{tabular}{|c|c|c|}
\hline $\begin{array}{c}\text { Acusto-optic } \\
\text { material }\end{array}$ & $\begin{array}{c}\text { Refractive } \\
\text { Index }\left(n_{0}\right)\end{array}$ & $\begin{array}{c}\text { Sound } \\
\text { velocity } \\
\left(\mathrm{ms}^{-1}\right)\end{array}$ \\
\hline Fused quartz & 1.46 & 5900 \\
\hline Tellurium glass & 2.10 & 3500 \\
\hline $\mathrm{As}_{2} \mathrm{~S}_{3}$ & 2.60 & 2600 \\
\hline $\mathrm{As}_{2} \mathrm{Se}_{3}$ & 2.89 & 2250 \\
\hline $\mathrm{KRS}-5$ & 2.57 & 1980 \\
\hline $\mathrm{KRS}-6$ & 2.30 & 1020 \\
\hline $\mathrm{PbMoO}$ & 2.38 & 3700 \\
\hline $\mathrm{NaBi}_{4}\left(\mathrm{MoO}_{4}\right)_{2}$ & 2.17 & 3950 \\
\hline $\mathrm{TeO}_{2}$ & 2.41 & 620 \\
\hline $\mathrm{GaP}_{3}$ & 3.31 & 6300 \\
\hline $\mathrm{TI}_{3} \mathrm{AsSe}_{3}$ & 3.33 & 1050 \\
\hline $\mathrm{LiNbO}_{3}$ & 2200 & 4100 \\
\hline
\end{tabular}




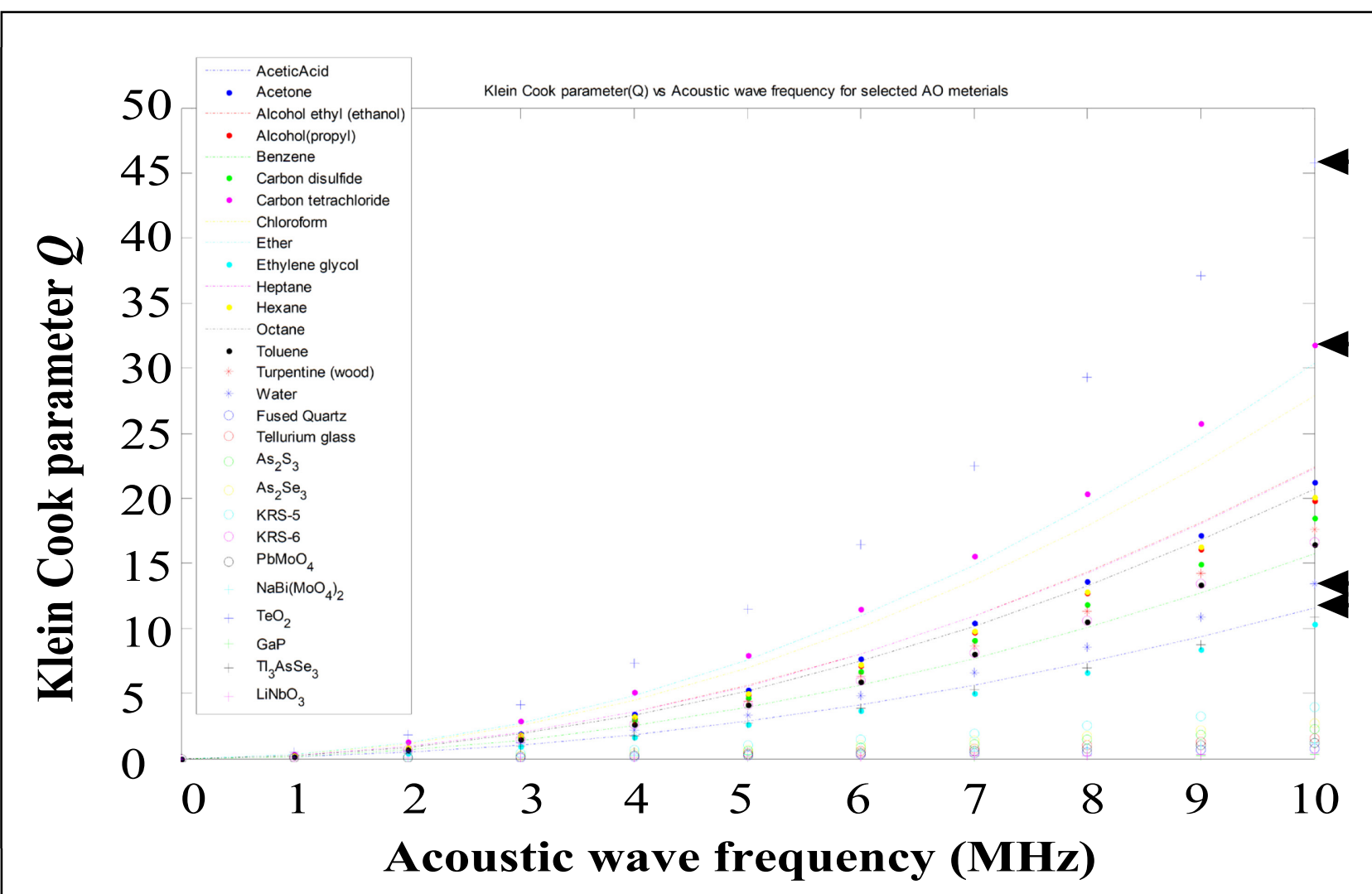

Figure 15. Graph of the parameter $\mathrm{Q}$ vs. Acoustic wave frequency liquids in table 1 and table 3 materials, with incident light wave length $\lambda=633 \mathrm{~nm}$ and acusto-optic interaction length $L=0.1 \mathrm{~m}$

\section{CONCLUSION}

The investigation of the ratio of diffraction light intensity for acusto-optic interaction in an two dimensional isotropic liquid with the assumption that there was no optical loss in the acoustic medium, for the optimum Klein Cook parameter $Q$ with incident light wave length of $\lambda=633 \mathrm{~nm}$, sound wave length of $\Lambda=0.1 \mathrm{~mm}$, acusto-optic interaction length $L=0.1$ $\mathrm{m}$, and the refractive index of the liquid in the range of 1 to 2 in Raman-Nath region showed that zeroth order has higher fraction of intensity compared to the other modes for the phase delay in the range 0 to 8 . The incident angle of light had no effect on the diffraction pattern in Raman-Nath regime and the energy was successively transmitted into large number of modes with zeroth order having the highest fraction compared to others with the same magnitudes of negative and positive diffraction orders overlapping. In non ideal Raman-Nath diffraction at $Q=10$ most of the incident light intensity was shared between zero and first orders. As the value of the Klein Cook parameter was increased, the energy distributions among the modes became unequal and the diffraction pattern approached Bragg regime. When Klein-Cook parameter was increased to 100, the diffraction pattern gave ideal Bragg diffraction. The highest $Q$ value for the liquids with refractive indices in the range 1.3 to 1.7 was obtained for carbon tetrachloride with a sound wave velocity of $926 \mathrm{~ms}^{-1}$ and a refractive index 1.46. Liquids having lower sound velocity have a higher $Q$ parameter for acusto-optic interaction. For acetic acid having a $Q$ value of 11, the diffraction pattern was in the Bragg diffraction regime for the phase delay range 0 to 5 . There is higher possibility of finding diffraction 
pattern in Bragg regime for a higher value of acoustic wave frequency. As acoustic wave frequency exceeded $10 \mathrm{MHz}$ most of the liquids reached Bragg regime and the crystals other than $\mathrm{TeO}_{2}$ which has the lowest sound velocity of $620 \mathrm{~ms}^{-1}$ needed higher acoustic wave frequency to reach Bragg regime.

\section{References}

[1] L. Brillouin, Ann. Phys. (Paris) 17 (1922) 88.

[2] C. V. Raman, N. S. N. Nath, Proc. Indian Acad. Sci. 4 (1936) 222.

[3] P. Phariseau, Proc. India Acad. Sci. 44A (1956) 165-170.

[4] W. R. Klein, B. D. Cook, IEEE Trans. Sonics Ultrasonic SU-14 (1967) 123.

[5] M. G. Moharam, L. Young, Applied Optics 17, (1978) 1757-1759.

[6] A. Korpel, Acusto-optics, (Applied Solid State Science, vol. 3, R. Wolfe, Ed, Academic Press, New York, 1972) ch. 2, p. 73.

[7] A. Korpel, T. C. Poon, Opt. Soc. Am. 70 (1980) 817.

[8] A.P.Goutzoulis, D.R.Pape, S. Kulakov (Edi), Design and Fabrication of Acousto-Optic Devices (Optical Engineering Vol. 41, Marcel Dekker, New York, 1994) .

[9] A. Korpel, R. Appel, M. G., J. Opt. Soc. Am. A10 (1993) 466.

[10] M. Born, E. Wolf, Principles of Optics (Cambridge (1959), 2002). 7th edition. 